\title{
La casa de Norman y Wendy Foster en Hampstead
}

\author{
Solé Bravo, Carlos \\ ETSAM-UPM, Departamento de Proyectos, Madrid, España, csolebra@gmail.com
}

\begin{abstract}
Resumen
Entre 1978 y 1979, Norman y Wendy Foster proyectan su propia vivienda en el barrio londinense de Hampstead. El proyecto más personal de los Foster permite - como en tantos otros casos- rastrear los referentes, obsesiones, sueños y frustraciones de sus autores.

Este proyecto representa el primer y único intento de aplicación en el campo de la arquitectura doméstica de un modelo, denominado por Reyner Banham "la nave bien servida", que utiliza la integración de sistemas como principal estrategia proyectual.

Los 18 meses de intenso trabajo reflejan, a través de las múltiples opciones desarrolladas, la evolución de la obra de la pareja de arquitectos, que avanza desde el funcionalismo fabril de la "nave bien servida", hacia el expresionismo tecnológico del denominado High-Tech.

En los motivos del fracaso del proyecto, que no llegaría a construirse, subyacen los debates y conflictos -entre arquitectura e ingeniería, prefabricación y artesanía, expresionismo y funcionalismo- en los que el estudio se encuentra inmerso a finales de los años 70 , y que precipitarán una nueva forma de entender la relación entre arquitectura y tecnología.
\end{abstract}

Palabras clave: Norman Foster, Wendy Foster, Nave bien servida, Integración de Sistemas, Reyner Banham.

\begin{abstract}
Between 1978 and 1979 Norman and Wendy Foster design their own home in the London neighbourhood of Hampstead. The most personal project of the Fosters allows us -as in many other cases - to trace the references, obsessions, dreams and frustrations of its creators.

This project represents a unique attempt to apply the "well-serviced shed" concept —as described by Reyner Banham - to the domestic realm. A concept based in the use of systems integration as the main project strategy. The 18 months of intense work show, through the various options developed, the evolution of the production of the couple of architects, from the industrial functionalism of the "well-serviced shed", towards the technological expressionism of the so-called High-Tech movement.

In the reasons behind the failure of this unbuilt project, one can read the debates and conflicts - between architecture and engineering, prefabrication and craftsmanship, expressionism and functionalism - in which the practice is immerse by the end of the 1970s. A series of events that will precipitate a new way of understanding the relationship between architecture and technology.
\end{abstract}

Key words: Norman Foster, Wendy Foster, Well-serviced shed, System integration, Reyner Banham. 
Innovación e Investigación en Arquitectura y Territorio. Departamento de Expresión Gráfica y Cartografía.

\title{
La casa de Norman y Wendy Foster en Hampstead
}

\author{
"Si construyes una casa para ti mismo es probable que se convierta en un experimento". \\ Serge Chermayeff
}

\section{Banco de pruebas}

La casa propia del arquitecto tiene la virtud de contener el universo de su autor, de descubrirnos sus referentes, sus pasiones y sus sueños. Tal vez esto baste para comprender el interés que suscita la casa que, entre 1978 y 1979, Norman y Wendy Foster proyectan en el exclusivo barrio londinense de Hampstead. Pero tras 18 meses de intenso trabajo, durante los que se elaboran hasta 8 opciones, con sus correspondientes bocetos, planos, maquetas e incluso prototipos estructurales a escala real y, habiendo iniciado su construcción, el proyecto es misteriosamente abandonado.

Una obra inconclusa, no construida - como la que nos ocupa - es una obra abierta, carente de decisiones finales, y desprovista de las limitaciones que la materia confiere a la arquitectura en el momento de su cristalización.

El proyecto de la casa de los Foster en Hampstead es, en realidad, muchos proyectos: la abundante documentación inédita, existente en los archivos de Foster + Partners, así como el testimonio directo de sus principales protagonistas, a través de conversaciones, permiten reconstruir la evolución del trabajo realizado. Un trabajo que no sigue un proceso lineal, sino que abre vías simultáneas de exploración, que evolucionan en paralelo al resto de la producción del estudio, nutriéndose de ella y, al mismo tiempo, contaminándola.

Pero esta casa, como cualquier proyecto no construido, esconde también la historia de una frustración. Prueba de ello es el hecho de que cuando, en 1979, la exitosa pareja de arquitectos británicos la incluye en su primera publicación monográfica de Foster Associates, la ocultan bajo el ambiguo título de "Housing system studies". Nada permite adivinar la verdadera naturaleza del proyecto más personal de los Foster, que se presenta como una obra huérfana, inacabada y sin emplazamiento. Ocupando las últimas páginas de la publicación, el proyecto parece destinado a anticipar el futuro del estudio, hacia una arquitectura de cada vez mayor sofisticación tecnológica.

Un breve texto explicativo la describe como un prototipo, un banco de pruebas, "un esqueleto estructural que se combina con una serie de unidades modulares intercambiables". ${ }^{2}$ Un proyecto nacido con la ambición de que exploraciones privadas encuentren aplicación posterior en edificios públicos. Recíprocamente, investigaciones previas del estudio en edificios públicos, son trasladadas por primera vez a la arquitectura doméstica.

Por su carácter experimental y por su firme voluntad de aplicación de la tecnología al ámbito doméstico, la residencia en Hampstead constituye un proyecto sin precedentes en la obra residencial de Norman Foster, cuyas anteriores incursiones en el campo de la arquitectura residencial marcan el inicio de su carrera con el Team Four. Las viviendas en Murray Mews (1963-1965), las casas Creek Vean en Cornwall (1964) y la Skybreak en Radlett (1965), representan interesantes desarrollos del "raumplan" loosiano, y denotan la influencia de los que fueran sus profesores en Yale: Paul Rudolph y Serge Chermayeff.

Pero los problemas de infiltración de agua, la mala calidad de los acabados y las dificultades en las uniones entre elementos, experimentados durante la construcción de dichas viviendas, propios de la imperfección de los sistemas constructivos tradicionales in situ, propiciaron la búsqueda de métodos alternativos que evitaran los inconvenientes derivados del uso de técnicas constructivas obsoletas. De ahí el interés por la utilización de sistemas prefabricados ligeros, listos para ser ensamblados en obra en el mínimo plazo de tiempo.

En su primera obra residencial construida con elementos prefabricados, el Bean Hill Housing (1971-73), reaparecen las nociones de privacidad, flexibilidad y adaptabilidad, enunciadas por Serge Chermayeff y Christopher Alexander en Community and Privacy. ${ }^{3}$ Aunque por motivos económicos se adopta un sistema de entramado estructural en madera, los arquitectos, en su afán de dotar al conjunto de una apariencia "tecnológica", revisten las fachadas de contrachapado con paneles ondulados de aluminio.

Por diversos motivos el proyecto sufrió modificaciones durante su construcción que, sumadas a las alteraciones introducidas a posteriori por sus habitantes, provocaron que el proyecto original terminara prácticamente irreconocible. Esta amarga experiencia, manifiesta la resistencia que el ámbito doméstico ofrece a la experimentación tecnológica pero, a la vez, representa un reto que los Foster se propondrán combatir en su propia vivienda.

\footnotetext{
${ }^{1}$ Serge Chermayeff, "A house is divided," House and Garden, mayo 1947: 96.

2 Foster Associates: Introduction by Reyner Banham (London: RIBA Publications, 1979$), 68$.

${ }^{3}$ Serge Chermayeff and Christopher Alexander. Community and Privacy: Toward a New Architecture of Humanism (London: Anchor Books, 1965).
} 
Este afán por crear una vivienda transformable, quizás tenga su origen en la experiencia de los Foster en Hampstead Hill Gardens: el pequeño apartamento, propiedad de Wendy, que serviría a la vez de residencia de la pareja de arquitectos y de oficinas del Team Four, desde su formación en 1963 hasta su disolución en 1967. Un apartamento-oficina, que cada mañana se transformaba en un estudio de arquitectura, en el que las visitas de clientes planteaban serios problemas de privacidad. El comedor se disfrazaba de sala de conferencias y la cama, oculta bajo grandes cajones de contrachapado, simulaba ser una mesa de reuniones.

No es hasta que el estudio crece, que los Foster se permiten el lujo de segregar vida doméstica y trabajo, primero en el estudio de Bedford Street, en Covent Garden (1969-1971), y posteriormente en Fitzroy Street (1971-1981), donde se fragua el proyecto de su vivienda en Hampstead.

\section{Kit de componentes}

Tal vez no sea casualidad que cuando, en junio de 1979, Ray Eames visita las oficinas de Foster Associates en Fitzroy Street, Norman y Wendy Foster están enfrascados en el proyecto de su casa. Ray acude a la ciudad para recibir la Medalla de Oro del RIBA, un tardío reconocimiento a la fructífera trayectoria junto a su marido, fallecido el año anterior. La citación del jurado, que cuenta con Norman Foster entre sus miembros, destaca la trascendencia de la famosa casa de los Eames en Santa Mónica. Considerada en su día el arquetipo del hogar moderno, ejerció una profunda influencia sobre toda una generación de arquitectos y, muy especialmente sobre los arquitectos británicos. Entre ellos Norman Foster quien, ya a principios de los años 60 , recién terminados sus estudios de postgrado en la Universidad de Yale, quedaría fascinado por la frescura de una arquitectura concebida a partir de componentes directamente sacados del catálogo Truscon, ensamblados en tan sólo día y medio, como si de un juego de Meccano se tratara.

No sorprende por tanto que diez años más tarde, en el segundo volumen de la monografía de Foster Associates, el propio Norman Foster —esta vez bajo el inequívoco título de "Foster Residence" - describa el proyecto de su casa en Hampstead en los siguientes términos: "la idea es crear un marco flexible, un marco que engloba el suelo, las paredes y el techo, para que en ese marco la planta sea completamente cambiable [...] la planta puede ajustarse a las circunstancias en la que nos encontramos como familia en cada momento en particular, respondiendo a patrones futuros que no podemos predecir". ${ }^{4}$ Una casa que, por tanto, concebida como un kit de componentes, es capaz de crecer y reconfigurarse respondiendo a las necesidades de sus usuarios.

Lejos de plantearse como un ejercicio teórico, el proyecto responde a un programa real y a un emplazamiento concreto: un terreno, de unos 1.600 metros cuadrados, que los Foster adquieren en Hampstead para construir una vivienda familiar, que planean habitar junto a sus dos hijos.

La elección de la parcela que, dado el importante desnivel existente, se encuentra flanqueada por altos muros de piedra que bloquean parcialmente la visión de la casa desde la calle, refleja la voluntad de privacidad de sus ocupantes y constituye el escenario idóneo para una edificación experimental, sin concesiones formales a una determinada ubicación ni a la arquitectura circundante.

Con acceso a través de un vaciado preexistente, la vivienda se adapta a la pendiente del terreno escalonándose, dando lugar a un porche semienterrado de hormigón, bajo el cual se sitúan el acceso y el aparcamiento. El volumen edificado, desarrollado principalmente en una sola planta, se posa sobre esta base de hormigón, como un contenedor ligero, caracterizado por la regularidad de su estructura portante expuesta al exterior y por la variedad de sus fachadas, en las que no es posible reconocer una composición de llenos y vacíos, sino un mosaico de cerramientos de distintas cualidades y texturas.

Contrastando con el carácter permanente de las viviendas georgianas y victorianas que predominan en el barrio, la casa ofrece un aspecto industrial, propio de una construcción despreocupada por cualquier responsabilidad representativa, en la que la envolvente, concebida como un sistema, surge como la respuesta adecuada de cada uno de los componentes a las necesidades de sus habitantes en cada momento.

La renuncia a la composición de las fachadas y la intercambiabilidad de sus componentes, convierten a esta vivienda en una obra sin precedentes en la producción del estudio. Los Foster están determinados a llevar al extremo la idea de edificio entendido como kit de componentes: el espesor de la fachada, determinado por el del armazón estructural, permitirá enchufar diversos tipos de cápsulas, que albergan aseos, estanterías, armarios, cocinas y diversos espacios de almacenaje. (Fig. 1)

\footnotetext{
${ }^{4}$ Norman Foster, Norman Foster: Works 1, ed. David Jenkins (London: Prestel, 2002), 483.
} 
Innovación e Investigación en Arquitectura y Territorio. Departamento de Expresión Gráfica y Cartografía. Arquitectura. Escuela Politécnica Superior. Universidad de Alicante. №2 julio 2014

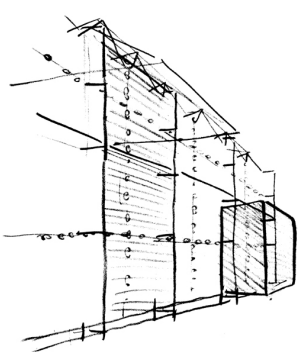

Fig. 1

Entusiasmado con la idea de diseñar hasta la última pieza de mobiliario, Norman Foster embarca a su equipo en un avión privado, que él mismo pilota rumbo a Paris, con el objetivo de visitar la Maison de Verre (1928-1932) de Pierre Chareau y Bernard Bijvoet: el paradigma de vivienda concebida como máquina de habitar. En palabras de Kenneth Frampton: "la planta transformable por excelencia [...] un laboratorio para el desarrollo de una hipotética arquitectura industrial". 5

Cautivados por la estética industrial del Self-contained Bathroom, patentado en 1927 por Bukminster Fuller para el núcleo central de servicio de su Dymaxion House, y por los componentes de la Maison Tropical en Niamey, diseñados por Jean Prouvé en 1949, los Foster pretenden aplicar el concepto de las cápsulas "enchufables" a su vivienda. Esa misma tarde Norman Foster y su séquito sobrevuelan el Mont Blanc en dirección a Bérgamo, donde visitan una fábrica de módulos de aseos de fibra de vidrio. El proyecto trata de cristalizar esa utopía propia del optimismo tecnológico de la Inglaterra de los años 60, que es la arquitectura del "plug-in". Un tema recurrente en las propuestas utópicas planteadas por Cedric Price en el Fun Palace (1961) o el Potteries Thinkbelt (1966), y por el grupo Archigram, en proyectos como la Plug-in City (196264), el Plug-in University Node (1965), las Plug-in Capsule Homes (1964) o las Gaskets Homes (1965). Obviando su reducido tamaño, cabría incluso encuadrar esta casa dentro de las Megaestructuras, descritas por Reyner Banham: "1 está constituida por unidades modulares, 2 es capaz de una ampliación grande y aún 'ilimitada', 3 es un armazón estructural en el que se pueden construir —o aun 'enchufar' o 'sujetar', tras haber sido prefabricadas en otro lugar - unidades estructurales menores, 4 es un armazón estructural al

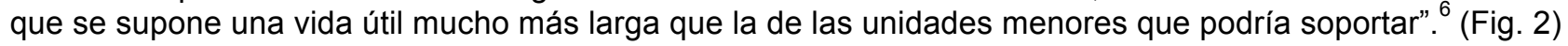

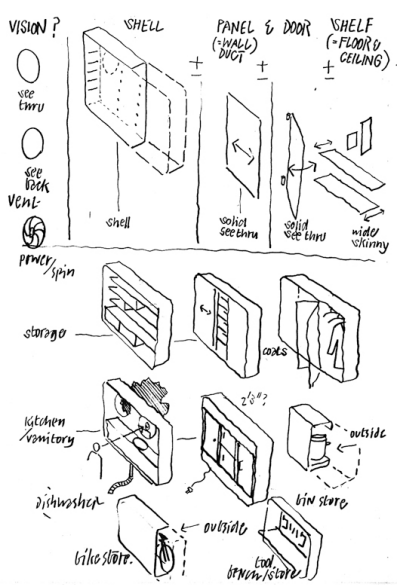

Fig. 2

\section{La nave bien servida}

La casa de los Foster en Hampstead representa el primer y único intento de aplicación a la vivienda unifamiliar, de un modelo, bautizado por el propio Banham como la "nave bien servida", iniciado con la fábrica para Reliance Controls Electronics en Swindon (1965-66), última realización del Team Four, tras cuya disolución será desarrollado con gran éxito comercial, tanto por Foster Associates, como por Richard Rogers, en multitud de edificios, principalmente destinados a empresas punteras en el campo de la investigación tecnológica. Destacan los edificios realizados por Foster Associates para Computer Technology: la Air-Supported Office (1969-1970) y la sede corporativa (1969-1971), ambas en Hemel Hempstead; y los realizados para IBM: las oficinas piloto en Cosham (1970-1971) y el IBM Technical Park

\footnotetext{
${ }^{5}$ Marc Vellay and Kenneth Frampton, Pierre Chareau: Architect and craftsman 1883-1950 (New York: Thames and Hudson, 1985$), 240$.

${ }^{6}$ Reyner Banham, Megastructure: Urban Futures of the Recent Past (London: Thames and Hudson, 1976), 8.
} 
Innovación e Investigación en Arquitectura y Territorio. Departamento de Expresión Gráfica y Cartografía. Arquitectura. Escuela Politécnica Superior. Universidad de Alicante. №2 julio 2014

en Greenford (1975-1980). La obra de Richard Rogers, tras la disolución del Team Four, representa una evolución paralela de la "nave tecnológica" de Norman Foster. Dadas las múltiples convergencias e influencias mutuas resulta difícil desvincular la obra realizada por ambos arquitectos durante los años 70.

En Theory and Design in the First Machine Age, Reyner Banham sitúa la llegada de la electricidad y posteriormente la de los electrodomésticos al hogar, como el punto decisivo en el desarrollo de la arquitectura moderna. Un privilegio inicialmente reservado a una élite y posteriormente al alcance de las masas: "ya hemos entrado en la Segunda Era de la Máquina, la era de los electrodomésticos, la química sintética, y podemos mirar atrás a la Primera, la era de las redes eléctricas y de la reducción de las máquinas a la escala humana, como un período del pasado."7

Banham sitúa los orígenes teóricos de esta arquitectura de la "Era de la Máquina", que se nutre del aprovechamiento de las transformaciones de la ciencia y la tecnología, en las propuestas teóricas nacidas en el seno de la École des Beaux-Arts en Paris, durante la segunda década del siglo XIX. Banham presenta a autores como Julien Gaudet, J. N. L. Durand y Auguste Choisy, como pioneros en la consideración del edificio como composición racional de elementos constructivos. La Histoire de l'Architecture, ${ }^{8}$ de Choisy, al que Le Corbusier se referirá como "el más valioso libro nunca escrito sobre arquitectura" ${ }^{9}$, da buena cuenta, a través de sus numerosas perspectivas analíticas de arquitecturas del pasado, de un modo de entender la evolución de la arquitectura, no como una sucesión de modas o estilos arbitrarios, sino como la consecuencia lógica de la evolución de los métodos constructivos disponibles en cada época.

Las características axonometrías explotadas realizadas durante los años 70 por el estudio de Norman Foster, ilustran esta aproximación a la arquitectura. Las espectaculares perspectivas de barcos, trenes y aviones, realizadas por John Batchelor para la revista Eagle y las secciones fugadas de Paul Rudolph —en cuyo estudio Norman Foster trabajará brevemente- representan dos claras influencias para esta forma de representación gráfica, que destripa la arquitectura, para mostrarla como el resultado de la integración de sus componentes.

Es precisamente en esta concepción de la arquitectura entendida a partir de sus elementos constituyentes, donde Reyner Banham encuentra la semilla que posibilitó el advenimiento del denominado Movimiento Moderno en los años 20. Si en la primera edición de su Theory and Design in the First Machine Age, Banham presenta a Buckminster Fuller como el verdadero continuador de esta tradición en el siglo XX, en su prólogo a la edición de 1980, no dudará en encumbrar a Norman Foster y Richard Rogers en la cúspide de la arquitectura de la "Era de la Máquina": "esas eclosiones de creatividad siguen proviniendo de hombres inspirados por el entusiasmo de la Era de la Máquina -uno piensa en los diseñadores del Centro Pompidou en París o el Sainsbury Centre en la Universidad de East Anglia. Pero esos entusiasmos son ahora plenamente cualificados; han perdido la inocencia de la amplitud de miras propia de los años 20 y 30 ."10

Tan deudora de la arquitectura californiana de las Case Study Houses, como de la Escuela de Alison y Peter Smithson en Hunstanton, con su exteriorización de estructuras e instalaciones, la "nave bien servida" consiste en su origen en un contenedor ligero, barato, de estructura prefabricada con grandes luces y cerramientos estandarizados, capaz de albergar actividades diversas en un espacio único, con capacidad para crecer y reconfigurarse cuando sus usuarios lo deseen. Un espacio en el que los conceptos de eficiencia, economía y prestaciones parecen anteponerse a consideraciones estéticas. Su origen fabril queda patente en el aspecto industrial de sus primeras realizaciones, consistentes en grandes contenedores neutros, con espacios interiores modulares, diáfanos, luminosos y flexibles, servidos por un suelo energético isótropo, en los que la distribución interior es confiada a particiones ligeras y piezas de mobiliario.

El Sainsbury Centre (1974-78), terminado el mismo año en que los Foster emprenden el proyecto de su casa, lleva la arquitectura de la "nave tecnológica" a su más alto grado de sofisticación. Se trata de un contenedor prismático alargado, que aloja las diversas actividades del centro de arte en un espacio único. Los pórticos en celosía de tubo de acero, que forman tanto las columnas como las vigas del edificio, salvan una luz de más de 30 metros, y alojan en su interior -en sus paredes y bajo el suelo- todas las instalaciones y los usos más permanentes del edificio. Esto permite dotar al espacio central de una gran flexibilidad, que permite la constante reorganización de la valiosa colección de arte moderno y precolombino de los Sainsbury, en un espacio diáfano con abundante luz cenital. (Fig. 3)

\footnotetext{
${ }^{7}$ Reyner Banham, Theory and Design in the First Machine Age (London: Architectural Press, 1960), 10.

${ }_{9}^{8}$ Auguste Choisy, Histoire de l'Architecture (Paris: G. Baranger, 1899).

${ }^{9}$ Le Corbusier, "La Modénature", 1925: 116.

${ }^{10}$ Reyner Banham, Theory and Design in the First Machine Age (Cambridge, MA: MIT Press, 1980), 10.
} 


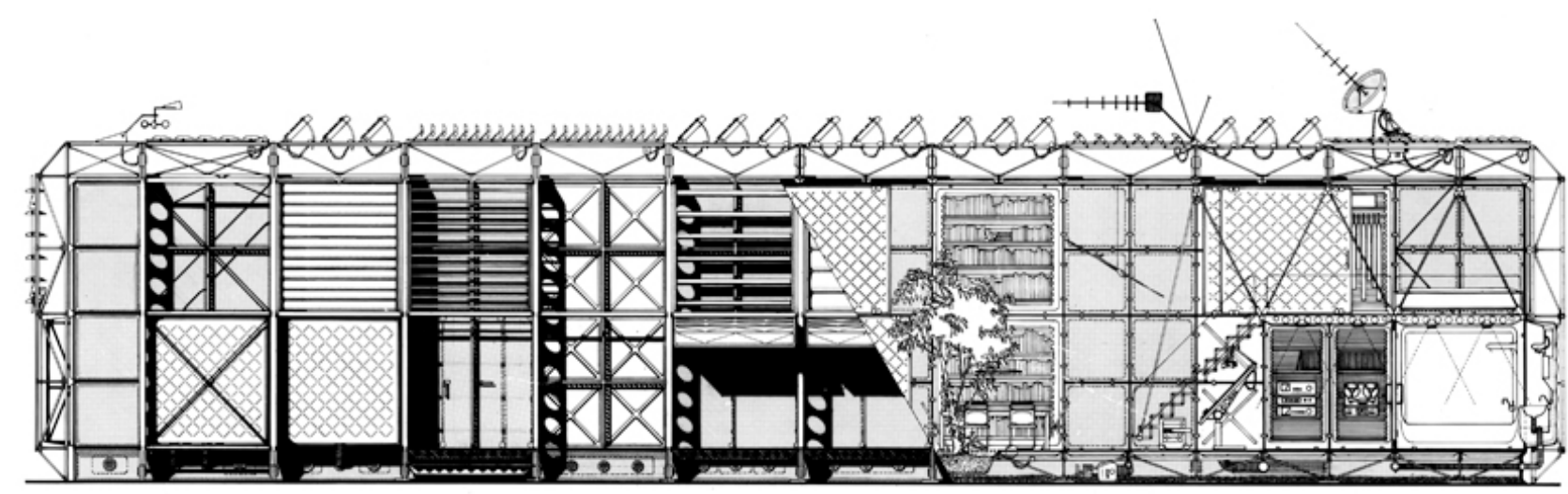

Fig. 3

\section{Integración de sistemas}

La estrategia proyectual encaminada a conseguir esta flexibilidad, consiste en la integración estratégica de los distintos sistemas que la componen: estructura, cerramientos, instalaciones, control solar, etc., en un edificio modular, en el que los elementos técnicos accesorios son trasladados al perímetro para liberar el espacio interior. Norman Foster afirma: "si observas los proyectos actuales de la oficina y estudias su historia ya sea un proyecto inicial como el Reliance Controls o uno posterior como el Aeropuerto de Hong Kong en Chek Lap Kok— te darás cuenta de que todos tratan sobre la integración". ${ }^{11}$ El concepto de integración adquiere, por tanto, en su obra, un doble significado:

Por un lado supone la convivencia de actividades y usos diversos bajo un mismo techo. De este modo, en la fábrica de Reliance Controls, la integración de producción y administración persigue la democratización del edificio fabril; el proyecto de la escuela de Newport, evita la segregación de las distintas actividades en edificios separados enfatizando el sentido de comunidad educativa; en las oficinas piloto de IBM el ordenador, tradicionalmente segregado, se disuelve por primera vez en el edificio de oficinas; en el Sainsbury Centre las zonas de exhibición, aprendizaje, administración y de esparcimiento comparten un mismo espacio, enriqueciendo tanto la experiencia del visitante como la del personal.

Por otro lado, el concepto de integración, constituye además un terreno mediador o intermedio, entre arquitectura y tecnología que, en palabras de Leonard R. Bachman, proporciona "un marco explícito para seleccionar y combinar los componentes del edificio de modo propositivo e intencionado". ${ }^{12}$ A propósito del Sainsbury Centre, Norman Foster afirma: "en este edificio el concepto de fachada y cubierta bicapa es alcanzado a través de la total integración de estructura, envolvente, iluminación e instalaciones -los elementos son interdependientes". ${ }^{13}$

Los trabajos de los arquitectos Richard D. Rush, The Building Systems Integration Handbook ${ }^{14}$ y Leonard R. Bachman, Integrated Buildings, constituyen intentos por establecer las bases de una teoría moderna sobre la integración de sistemas en arquitectura. Siguiendo la tradición iniciada por Auguste Choisy, ambos autores recurren al análisis de numerosos ejemplos contemporáneos, apoyándose en la representación gráfica y en el uso de numerosos diagramas disectivos.

Chermayeff y Alexander coinciden con este análisis cuando afirman que "el problema debe fragmentarse de acuerdo con sus intersticios. La mayoría de las partes de un problema están tan estrechamente vinculadas entre sí que de nada sirve considerarlas en forma independiente. Debemos intentar hallar partes que se configuren con tanta integridad que puedan ser consideradas como unidades aisladas". ${ }^{15}$

Se trata de analizar la complejidad del edificio a través de una visión integral del conjunto, lo cual, como observaba Le Corbusier, convierte al arquitecto en un organizador: "la arquitectura es organización. Usted es un organizador y no un estilista de tablero de dibujo". ${ }^{16}$ El elevado nivel de sofisticación técnica requerido para la consecución de los proyectos del estudio exige, desde las primeras etapas de diseño, el trabajo conjunto de un equipo pluridisciplinar, en el que el arquitecto asume el papel de coordinador. Como observa Leonard R. Bachman, "lo que en un principio evolucionó en una organización jerárquica con el arquitecto en la cumbre, ha resultado en una red profundamente interconectada de información y decisiones compartidas."17

\footnotetext{
${ }_{11}^{11}$ Norman Foster, Norman Foster: Works 1, ed. David Jenkins (London: Prestel, 2002), 119.

${ }_{12}^{12}$ Leonard R. Bachman, Integrated Buildings: The Systems Basis of Architecture (Hoboken: John Wiley \& Sons, 2003), 4.

${ }^{13}$ Norman Foster, "Norman Foster: Interview," entrevistado por Marc Emery, L'Architecture D'Ajourd'hui, no. 243, febrero 1986: LVIII.

${ }^{14}$ Richard Rush, The Building Systems Integration Handbook (New York: John Wiley \& Sons, 1986).

${ }^{15}$ Serge Chermayeff and Christopher Alexander. Community and Privacy: Toward a New Architecture of Humanism (London: Anchor Books, 1965), 170.

${ }_{16}^{16}$ Le Corbusier, "Si tuviese que enseñarles arquitectura", Architectural Design, vol. 29, febrero 1959.

${ }^{17}$ Leonard R. Bachman, Integrated Buildings: The Systems Basis of Architecture (Hoboken: John Wiley \& Sons, 2003$), 6$.
} 
Innovación e Investigación en Arquitectura y Territorio. Departamento de Expresión Gráfica y Cartografía. Arquitectura. Escuela Politécnica Superior. Universidad de Alicante. №2 julio 2014

Así, el equipo de diseño de la casa de los Foster en Hampstead, además de Norman y Wendy Foster, cuenta con la presencia de los habituales arquitectos e ingenieros "especialistas" del estudio: los arquitectos Tony Pritchard, Richard Horden, Jan Kaplický y Peter Busby, y los ingenieros Loren Butt (instalaciones) y Anthony Hunt (estructuras). Cabe destacar el papel de este último quien, apodado "Meccano Man" por Peter Cook, será el artífice de todas las estructuras proyectadas por Norman Foster desde la época del Team Four. Las estructuras ligeras de Hunt, concebidas como un kit de componentes, optimizadas para erigirse en el mínimo tiempo y con el menor coste, y su capacidad de comunicación con los arquitectos desde las primeras fases del diseño, facilitaron este trabajo pluridisciplinar que posibilitó el desarrollo de la "nave bien servida".

Los precedentes de la integración de sistemas como estrategia proyectual se hallan principalmente en Estados Unidos: en las realizaciones de la Escuela de Chicago, en las que el uso de estructuras metálicas favoreció la vinculación de los sistemas de ventilación e iluminación a un incipiente cerramiento en muro cortina; así como en los edificios de Frank Lloyd Wright -que Norman Foster estudiaría a través de In the Nature of Materials, de Henry-Russell Hitchcock- ${ }^{18}$ como el Larkin (1906) en Búfalo, con su integración de sistemas ambientales y mobiliario industrializado, o el Johnson Wax Administration Center (1939) en Racine, Wisconsin, con su organización unitaria de los empleados y la integración de sistemas de acondicionamiento ambiental.

Esta concepción integral del proyecto, está asimismo presente en la obra de Buckminster Fuller, mentor y colaborador de Norman Foster, para quien "un diseñador es la síntesis emergente de artista, inventor, mecánico, economista y estratega". 19 En la futurista casa Dymaxion, adaptando estructuras ligeras propias de la industria aeronaval, Fuller plantea una vivienda en forma de anillo hexagonal suspendida de un mástil central que aglutina sus servicios mecánicos.

La característica articulación formal de espacios servidores y servidos, propia de las realizaciones de Louis Kahn en colaboración con el ingeniero August Komendant, es palpable en obras como el Richards Medical (1959) en Filadelfia, cuyas torres de servicio integran ventilación, estructura y núcleos de comunicación, o el Museo Kimbell (1967) en Texas, con sus bóvedas de hormigón que integran estructura, iluminación natural y ventilación. A pesar de que en 1961, Louis Kahn impartía clases en la universidad de Pensilvania, su influencia en la universidad de Yale, era palpable. No solo a través de sus aportaciones como profesor invitado, sino también a través de su edificio para la Galería de Arte, terminada en 1953, en cuyo piso superior Norman Foster, Richard Rogers, junto a otros once estudiantes, trabajarían día y noche a las órdenes de Paul Rudolph. Un edificio repleto de innovaciones tecnológicas, en el que por ejemplo, la estructura espacial tetraédrica actuaba a su vez de conducción de aire.

Pero serán las investigaciones llevadas a cabo por Ezra Ehrenkrantz, primer teórico de la integración de sistemas, las que ejerzan una influencia directa en la "nave bien servida" de Norman Foster. En particular a través del School Construction Systems Development (SCSD), un programa iniciado en Inglaterra y trasladado a los Estados Unidos a principios de los años 60, destinado a la provisión de edificios escolares eficientes y de bajo coste. Frente a los sistemas cerrados de prefabricación —como los de Marcel Breuer, Walter Gropius y Konrad Wachsmann, entre otros- cuyos componentes están predeterminados, quedando el arquitecto obligado a desarrollar su vocabulario específico, Ehrenkrantz propone sistemas abiertos, cuyos elementos integrantes -estructuras, instalaciones, particiones, etc.- son compatibles con otros sistemas así como con productos convencionales de construcción. ${ }^{20}$ Durante su estancia en Estados Unidos, Norman Foster y Richard Rogers quedarán fascinados por este prototipo, cuya influencia se dejará notar en su propuesta para el concurso de la Escuela en Newport, de 1965.

Los elegantes edificios modulares del suizo Fritz Haller, en particular su fábrica USM en Münsingen (1962), anterior a la fábrica de Reliance, representan el que tal vez sea el primer ejemplo europeo de integración de sistemas. Haller desarrolló, durante los años 70 y 80 , tres sistemas constructivos en acero, denominados "Stahlbausystem", compuestos por estructuras en celosía o vigas aligeradas sobre esbeltas columnas, organizados por tamaños en función de su uso: el sistema Mini”, con el que construyó su propia vivienda, "Midi”, para escuelas y oficinas, y "Maxi", para fábricas. Estos elegantes sistemas modulares, al igual que su exitoso sistema de mobiliario USM, están integrados por un limitado número de componentes que, gracias a un versátil sistema de conexión, permite múltiples configuraciones. La influencia de la arquitectura de Haller sobre la obra de Norman Foster, pese a que nunca ha sido documentada, se evidencia en la estrecha relación mantenida por el suizo con Anthony Hunt, así como por la reconocida admiración de Norman Foster hacia el sistema de mobiliario USM, utilizado recurrentemente en sus proyectos personales. (Fig. 4)

\footnotetext{
${ }^{18}$ Henry-Russell Hitchcock, In the Nature of Materials: The Buildings of Frank Lloyd Wright 1887-1941 (Ann Arbor, MI: Da Capo Press, 1942).

${ }^{19}$ Richard Buckminster Fuller, Ideas and Integrities: A Spontaneous Autobiographical Disclosure (New York: Collier, 1969), 109.

${ }^{20}$ Ezra D. Ehrenkrantz, Architectural Systems: A needs, Resources, and Design Approach (New York: Mc Graw-Hill, 1989 ), 2.
} 


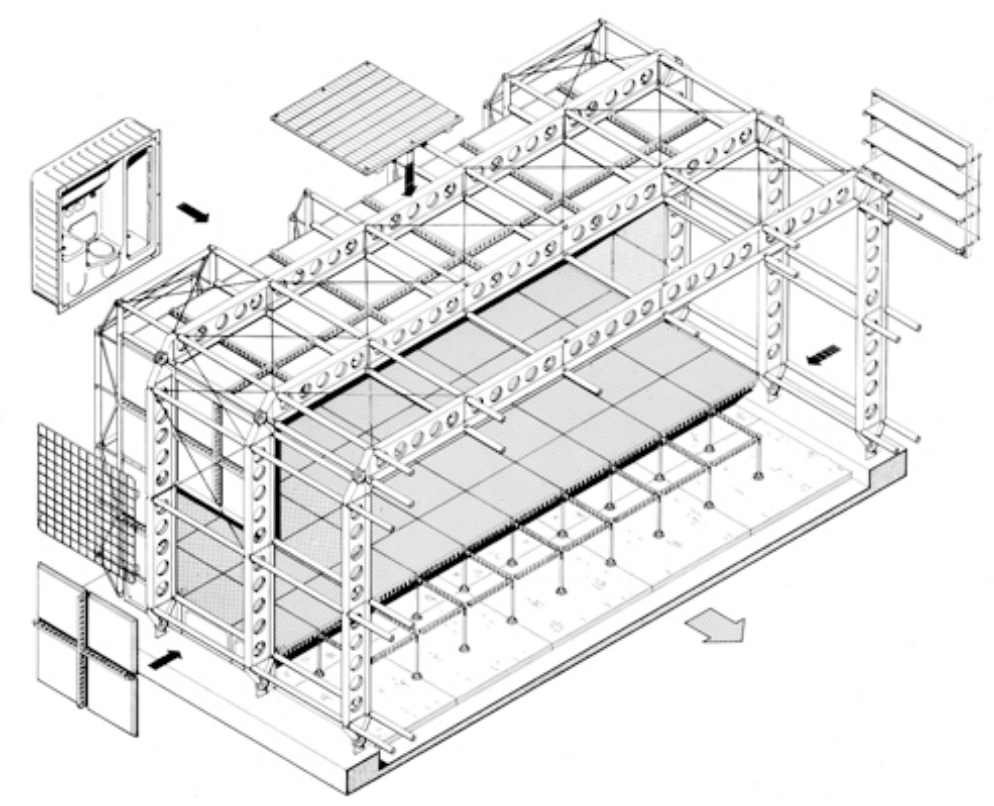

Fig. 4

\section{Evolución de una idea}

Del mismo modo que los planteamientos introducidos por la "nave bien servida" ofrecen la oportunidad de redefinir tipologías establecidas, la "casa tecnológica" en Hampstead constituye un laboratorio de ensayo, no solo de nuevos materiales y sistemas constructivos, sino también de nuevas formas de habitar. Como afirmaba Serge Chermayeff, "nuestra habilidad para fabricar un continente plástico del tamaño de una casa y llenarlo luego de adminículos mecánicos difícilmente puede señalarse como un progreso arquitectónico real si la organización entera sigue respondiendo a objetivos anticuados". ${ }^{21}$

Si como escribe Deyan Sujdic, "todas las casas que Foster ha construido estaban cuidadosamente diseñadas para indicar algo muy concreto sobre el tipo de arquitecto que él quería ser en diversos momentos de su vida", ${ }^{22}$ la casa en Hampstead - su primer proyecto de vivienda propia- expresa, mejor que ninguna otra obra, el entusiasmo de la pareja de arquitectos por las posibilidades ofrecidas por la tecnología en la "era de la máquina". La evolución del proyecto, desde sus primeras versiones en acero, hasta las complejas soluciones en aluminio, revela la creciente importancia concedida a la expresión de la estructura, a la integración de los servicios mecánicos y a la intercambiabilidad de los componentes.

Los primeros planos de la vivienda, delineados por el propio Norman Foster en julio de 1978, destinados a obtener permiso de obras, muestran un volumen prismático que, apoyado sobre el lindero con el vecino, se articula a lo largo de dos patios lineales, segregando tres pabellones: el dormitorio principal y la sala de estar, los dormitorios de los hijos y una cocina comedor, y un dormitorio de invitados.

Se trata de un esquema derivado de los modelos propuestos por Serge Chermayeff y Christopher Alexander en Community and Privacy, y claramente influido por la casa propia de Serge Chermayeff en Connecticut, New Haven. Chermayeff entiende el patio como una habitación exterior, destinada no sólo a mediar entre la naturaleza y la vida doméstica sino también a jerarquizar distintos grados de privacidad visual y acústica dentro de la vivienda. Su preocupación, casi obsesiva, por la privacidad en el ámbito doméstico, queda patente cuando declara: "la vivienda es un pequeño entorno, y dentro de él se están introduciendo cada vez más profundamente las tensiones y desgastes que hoy corroen al mundo exterior. Para servir a los intereses de la privacidad, dos de estas tensiones en particular, el tránsito y el ruido, deben ser tratadas como si fueran invasores". ${ }^{23}$

Ya en esta primera propuesta, los elementos accesorios como armarios y aseos, reducidos a su mínima expresión, son desplazados al perímetro, proyectándose hacia el exterior. Los Foster llevan al extremo la idea de la segregación del dominio de las máquinas y el dominio de las personas. Una idea enunciada en 1963 por Serge Chermayeff y Christopher Alexander: "la zonificación funcional de la vivienda depende de

\footnotetext{
${ }^{21}$ Serge Chermayeff and Christopher Alexander. Community and Privacy: Toward a New Architecture of Humanism (London: Anchor Books, 1965), 66.

${ }_{22}$ Deyan Sudjic, Norman Foster: A Life in Architecture (London: Weidenfeld \& Nicolson, 2010), 241.

${ }^{23}$ Serge Chermayeff and Christopher Alexander. Community and Privacy: Toward a New Architecture of Humanism (London: Anchor Books, 1965), 34
} 
una separación adecuada de los dominios socialmente definidos"; ${ }^{24}$ y que Charles Moore, Gerald Allen y Donlyn Lyndon retomarían en 1974: "es útil concebir los espacios que rodean las máquinas, no como habitaciones sino como 'dominios de las máquinas'. Las máquinas y sus dominios existen para servirnos en nuestra casa, no para dominarnos, y nos sirven mejor cuando no infringen el fin más general de las habitaciones". ${ }^{25}$

Frente a los refugios casi militares de Buckminster Fuller: Dymaxion (1929) y Wichita (1944), cuya rigidez espacial es fruto de su organización alrededor de un núcleo central eficiente que integra estructura, instalaciones y todo el equipamiento fijo de la vivienda, los Foster proponen un esquema descentralizado, que permite liberar el centro de la casa de todo aquello que pueda estorbar la flexibilidad del espacio interior.

La estructura sigue un rígido esquema modular de montantes y travesaños de acero, coronados por una malla espacial, similar a la del Sainsbury Centre, parcialmente oculta desde el exterior tras los paneles de protección solar.

En la siguiente versión, la planta evoluciona hacia una forma más compacta, con el aparcamiento semienterrado. Un gran contenedor diáfano en el que un núcleo cerrado contiene el programa más privado de la vivienda. La estructura, con sus cruces de San Andrés y las triangulaciones de la cubierta, se expresa claramente al exterior. El muro-biblioteca en dos niveles, denota de nuevo la influencia de la Maison de Verre.

En la tercera versión la casa se separa del vecino. El contenedor diáfano, está formada por pórticos bidireccionales que, de manera análoga al Sainsbury Centre, integran tanto la cubierta como los elementos verticales. (Fig. 5)

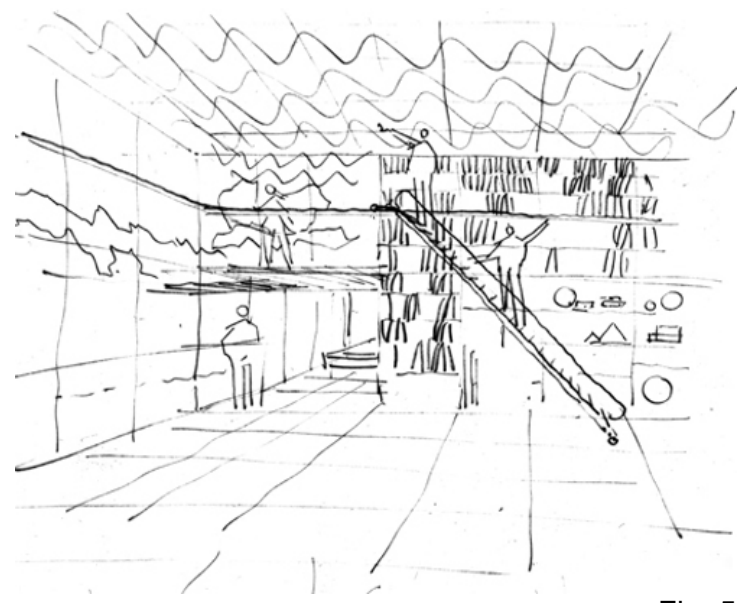

Fig. 5

Una de las versiones de la casa que mayor interés suscitó en el estudio, fue la desarrollada por Richard Horden, cuya planta presenta una geometría indeterminada, de contornos quebrados, formada por la concatenación de 16 módulos idénticos de 4,8 x 4,8 metros. El espacio interior resultante, compuesto por la agrupación de estas unidades modulares cuadradas, es un espacio fluido sin pasillos ni jerarquías espaciales, en el que las distintas actividades se separan mediante ligeras particiones correderas y pivotantes, capaces de establecer diversos grados de relación entre zonas de ocio, descanso y trabajo en función de las exigencias de cada momento.

\footnotetext{
${ }^{24}$ Ibid., 235.

${ }^{25}$ Charles Moore, Gerald Allen and Donlyn Lyndon, The Place of Houses (New York: Henry Holt \& Co, 1979), 79.
} 


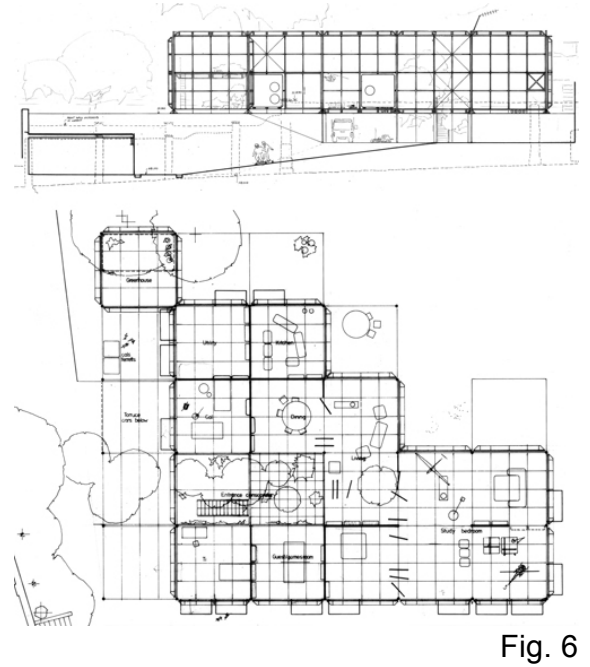

Una idea que remite a la idea de Louis Kahn, de la casa concebida como sociedad de estancias: "la arquitectura nace de la construcción de una estancia... la estancia es un lugar de la mente... la planta es una sociedad de estancias. Un buen lugar para vivir, trabajar, aprender...." ${ }^{26}$ Y también a su empleo del cuadrado como unidad fundamental: "siempre empiezo con cuadrados, no importa de qué problema se trata”. ${ }^{27}$ La serie de proyectos residenciales que Kahn realiza en los años 50 -la casa DeVore en Montgomery (1954-1955), la casa Adler en Filadelfia (1954-1955) y la casa de baños en Trenton (1954-59)se basa en el uso del cuadrado como unidad espacial. La habitación se reduce a su forma geométrica más elemental, eliminando cualquier impureza programática. De este modo la casa queda dividida en sus actividades más esenciales: lugares para comer, para dormir, para disfrutar la compañía de otros, para cocinar, cada una con su propio cuadrado asignado. Pero a diferencia de la vivienda de los Foster, cuya apariencia es siempre unitaria, cada uno de estos módulos -que Kahn denomina "clusters"- constituyen unidades autónomas, con estructura propia, y que, por tanto, se expresan al exterior como volúmenes diferenciados.

Por otra parte, el propio Kahn escribe: "hoy debemos construir con piedras huecas... La naturaleza del espacio está caracterizada por los espacios menores que lo sirven. Las habitaciones de almacenaje, servicios y cubículos no deben ser áreas segregadas dentro de una estructura espacial unitaria, sino que deben disponer de su propia estructura" ${ }^{28}$ Una idea que ejemplifica su particular visión de la ya comentada integración de sistemas, basada en la utilización de la estructura portante como elemento definitorio de espacios "servidores y servidos". En la vivienda de los Foster sin embargo, los espacios servidores no se alojan como en Kahn en el espesor de pilares interiores sino que se confinan a la periferia: a las oquedades de la estructura perimetral y al interior del suelo técnico.

Richard Horden propuso a los Foster para esta versión de la casa, una solución estructural modular compuesta por un entramado en aluminio con esbeltas columnas circulares. Anthony Hunt -quien había participado ese mismo año, junto a Michael Hopkins (ex-socio de los Foster) en el SSSALU (Short Span Structures in Aluminium), un sistema modular en aluminio, que fracasó comercialmente por su elevado costo- desaconsejó expresamente el uso de aluminio, por la imposibilidad de utilizar perfiles estándar, y por la dificultad en la ejecución de los nudos. Sin embargo Norman Foster, empeñado en emplear el material más ligero posible, prefirió seguir explorando esta vía.

En 1983, habiendo abandonado el estudio de Foster Associates, Richard Horden, diseñó y construyó junto al ingeniero de estructuras Frank Newby, la Yacht House en New Forest. Una vivienda modular, basada en el sistema propuesto inicialmente a los Foster para su casa -y en los detalles Rafael Soriano y Craig Ellwood para las Case Study Houses- utilizando secciones de mástil de aluminio como vigas, apoyadas sobre delgadas columnas tubulares también de aluminio. Esta vivienda, de presupuesto limitado y que, en gran parte, fue auto-construida, fue visitada por los Foster en el momento de su terminación.

En las versiones de octubre y noviembre de 1978, Richard Horden desarrolla otra opción aún más compleja estructuralmente, con pórticos bidireccionales formados por perfiles alveolados en aluminio en la dirección transversal, y elementos en celosía en la longitudinal, para soportar una cubierta cuya compleja malla espacial queda totalmente expuesta al exterior.

\footnotetext{
${ }^{26}$ Louis Kahn, The Room, 1971. Carboncillo sobre papel amarillo, publicado en: Yutaka Saito, Louis I. Kahn Houses (Tokyo: Toto, 2004$), 27$.

${ }^{27}$ Yutaka Saito, Louis I. Kahn Houses (Tokyo: Toto, 2004), 23.

${ }^{28}$ Ibid.
} 
El uso de los perfiles alveolados, empleados en las primeras propuestas para el Sainsbury Centre y en la Fábrica Renault, denota la fascinación de Norman Foster por la industria aeronaval. El propio Norman Foster reconoce la influencia, en el proyecto de su casa, de las cabinas de los dirigibles de los años 30 , concebidas para obtener la máxima ligereza.

El espacio diáfano de la vivienda está subdividido mediante particiones ligeras pero, a diferencia de las versiones anteriores, los núcleos de servicio se disponen a lo largo de una espina central, dando lugar a una sección que recuerda la de los refugios diseñados por Jean Prouvé para la Maison Tropical en Niamey, Níger (1949). La envolvente está caracterizada por la alternancia de paneles acristalados y sólidos, y por la potente expresión de la estructura, para la que se erigieron prototipos a tamaño real. (Fig. 7)

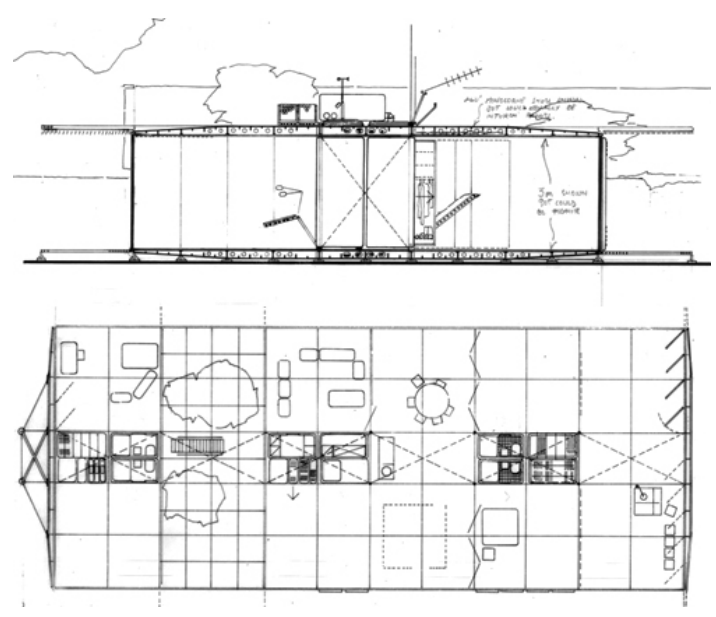

El exhibicionismo estructural de las últimas versiones de la casa, parecen remitirnos a la Crystal House de George Fred Keck, realizada para la exposición de Chicago de 1934. Un enorme prototipo residencial compuesto por una aparatosa estructura en celosía, que Norman Foster conocería a través de Buckminster Fuller, quien se fotografiaría junto a su Dymaxion Car en frente del edificio.

La versión más elaborada, de principios de 1979, fue la que logró mayor atención por parte de los Foster. Ésta representa un desarrollo de los esquemas anteriores en aluminio. El esqueleto principal está formado por pórticos formados por pletinas de aluminio con alveolos circulares. Una celosía de pórticos de aluminio con tensores, perpendicular a los anteriores, actúan de arriostramiento. Los nudos articulados, se resuelven mediante complejas inserciones de acero, que aseguran la correcta transmisión de esfuerzos.

Este entramado estructural permite la fijación de un número ilimitado de paneles prefabricados de fachada: translúcidos, transparentes, con mayor o menor grado de aislamiento, todos ellos intercambiables, con la única restricción de que éstos deben adoptar el módulo básico de 1,2 metros. Esta idea se extiende también a la cubierta, en la que se alternan zonas abiertas, opacas y transparentes, estas últimas en forma de grandes lucernarios acristalados con elementos de protección solar. (Fig. 8)

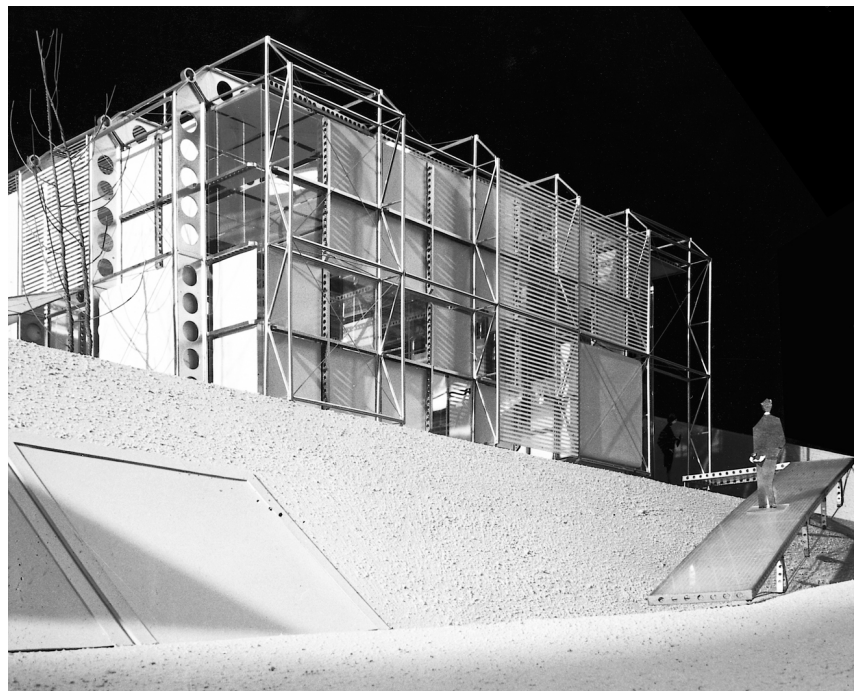


Innovación e Investigación en Arquitectura y Territorio. Departamento de Expresión Gráfica y Cartografía. Arquitectura. Escuela Politécnica Superior. Universidad de Alicante. №2 julio 2014

ISSN: 2341-0515

Fig. 8

Además, la sección de los perfiles se moldea en forma ovalada para facilitar la fijación de un sinfín de componentes auxiliares, tales como bombas de calor, lamas, persianas, aleros retráctiles de control solar, paneles móviles de aislamiento, paneles solares y equipos de alumbrado exterior. Tal como muestran los dibujos elaborados por Richard Horden y Jan Kapliký, la intercambiabilidad de los componentes confiere al conjunto un aspecto indeterminado, en constante transformación.

Las cápsulas de servicio, que incluyen aseos, estanterías, armarios, cocina, papeleras y todo tipo de zonas de almacenaje, son ahora verdaderos componentes plug-in, capaces de alojarse en las oquedades de la estructura de fachada, para lograr una planta completamente diáfana. La distribución de la vivienda ya no responde a los esquemas propuestos por Chermayeff y Alexander. El patio, confinado a una esquina de la vivienda, al que se accede a través de una escalera-rampa, actúa ahora como vestíbulo distribuidor.

La sección transversal revela la importancia del mobiliario que, dada la indefinición formal de las estancias, constituye el único elemento que cualifica el espacio. Los sillones del finlandés Yriö Kukkapuro, utilizados en el Sainsbury Centre, las sillas de los Eames, la lámpara Paréntesi de Achile Castiglioni, las maquetas de aeroplanos suspendidas de la cubierta y los modelos de helicópteros sobre las estanterías, constituyen referentes, para una casa que pretende erigirse como un manifiesto tecnológico doméstico, para el que la eficiencia, la ligereza y la flexibilidad son parámetros fundamentales. (Fig. 9)

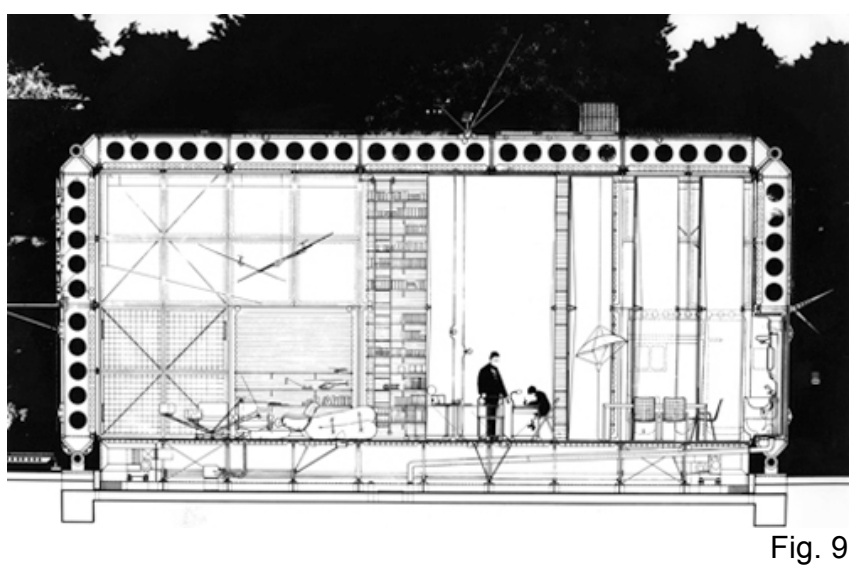

\section{6. ¿Por qué no construyó su casa, Sr. Foster?}

En la introducción a la mencionada monografía de Foster Associates, el teórico Reyner Banham, con su habitual euforia tecnocrática sentencia: "construir Arquitectura Moderna [...] es practicar la arquitectura del riesgo". ${ }^{29}$ Indagar los motivos del fracaso de la casa de los Foster tal vez nos permita ahondar en la naturaleza de dicho riesgo. Valorar su alcance y sus consecuencias.

\subsection{El banco de Hong Kong}

El texto de la mencionada monografía justifica el abandono del proyecto por "la fuerte carga de trabajo que supuso el inicio del Banco de Hong Kong". En agosto de 1979, el equipo de Foster Associates participa en el concurso para el Banco de Hong Kong and Shanghai, en Hong Kong, proyecto que nacido bajo el eslogan de "el mejor edificio bancario del mundo", marca un punto de inflexión en la producción de Foster Associates.

A pesar de que el interés por la tecnología y la integración persisten en la arquitectura del Banco de Hong Kong, las diferencias con los proyectos anteriores del estudio son notables. La magnitud y la complejidad del encargo, hacen que los Foster prescindan de sus habituales colaboradores, incluyendo a Anthony Hunt para quien la casa en Hampstead será la última colaboración con Foster Associates- para contratar los servicios de una firma internacional como Arup Associates, y de numerosos técnicos especialistas. Este cambio organizativo provocará un creciente distanciamiento entre arquitectura e ingeniería, que desembocará en una disociación entre arquitectura y tecnología.

Una disociación que queda patente en el hecho de que el empleo de sofisticados componentes industriales, no se justifica ya desde criterios de eficiencia y economía, sino que está destinado exclusivamente a garantizar el control estético del producto final. Los Foster retoman el concepto de cápsulas "enchufables",

\footnotetext{
${ }^{29}$ Reyner Banham, introducción a Foster Associates: Introduction by Reyner Banham (London: RIBA Publications, 1979$), 4$.
} 
ensayado en su casa, introduciendo en los núcleos de servicio del banco, enormes módulos metálicos prefabricados, destinados a alojar aseos, unidades de aire acondicionado y zonas de almacenamiento. Módulos que, una vez agrupados y revestidos mediante una piel continua de acero inoxidable, son totalmente fijos.

Los componentes, producidos en países distintos, están diseñados y fabricados a medida para el edificio, como si de las piezas de un avión se tratara, por lo que, cuando se terminó, 7 años más tarde, el mejor edificio bancario del mundo resultó ser también el más caro. El plug-in se convierte en un mero gesto, el edificio no es ya una megaestructura, sino un auténtico monumento a la tecnología. Al servicio de las aspiraciones comerciales de una gran corporación, el proyecto confirma las palabras de Reyner Banham cuando advierte: "en la sociedad abierta de miras, con su movilidad social e individual, su intercambiabilidad de componentes y personal, su aparataje, y su casi universal desechabilidad, la persistencia de la arquitectura como espacio monumental debe tomarse como evidencia del valor sentimental de lo perdurable." 30

Tras resultar ganadores del concurso, el estudio crece y se internacionaliza. A caballo entre dos modos de entender la arquitectura, la propia evolución del proyecto en Hampstead es un reflejo de como el funcionalismo tecnológico de la arquitectura de la "nave bien servida", cuyo máximo exponente en el Sainsbury Centre, da paso al expresionismo heroico-tecnológico del banco de Hong Kong (1979-1986), paradigma de la arquitectura High-Tech.

\subsection{Un vecino entrometido}

El propio Norman Foster, interrogado acerca de los motivos por los que no construye su casa, añade al anterior argumento los problemas con el vecino: "siempre tuvimos el problema del vecino. El vecino era una persona difícil. Nunca nos sentimos realmente cómodos con el vecino". Esto explica el progresivo distanciamiento de la edificación del lindero con la parcela del Sr. Greenway, hasta el punto de renunciar a una óptima orientación del jardín, al adosar la casa al lindero opuesto.

Resulta curioso observar como los Foster se convierten en víctimas de un problema que Chermayeff identificaría como propio de la casa suburbana: "la pseudo-casa de campo, mal ubicada dentro de un conjunto mal organizado, no está en íntimo contacto con la del vecino, ni tampoco lo bastante alejada de ella; sus flancos están desprotegidos contra las miradas curiosas y contra los ruidos molestos [...] El vecino continua siendo un extraño y los verdaderos amigos, con frecuencia, viven lejos [...] El vecino de al lado, con gustos distintos de los nuestros que se expresan a menudo mediante sonidos estridentes, se ha transformado repentinamente de vecino deseable en extraño entrometido". ${ }^{31}$

\subsection{Wendy Foster}

Richard Horden menciona el diagnóstico de un cáncer a Wendy Foster como una posible causa del abandono de la vivienda. Fallecida en 1989, poco se sabe acerca del papel desempeñado por Wendy Foster (de soltera Wendy Cheesman) en el estudio. Su figura siempre ha quedado en un segundo plano, ensombrecida tras la imagen dominante de su marido, pese a que todos sus antiguos colaboradores del estudio subrayan la importancia de su papel en aquellos años. Wendy siempre estuvo presente en cada reunión del equipo de diseño, participando activamente junto a su marido en la toma final de decisiones, así como en la organización del estudio.

En 1963, recién graduada por el Regent Street Polytechnic de Londres, Wendy destaca por la elegancia de sus dibujos a lápiz y por su sofisticada aproximación al diseño, por lo que su amigo Richard Rogers, propone a Norman Foster incorporarla a su equipo. De familia acomodada, hija de un antiguo director de Lloyds, la familia de Wendy ofreció al Team Four algunos de sus primeros encargos, como la ampliación de la casa de la madre de Wendy en Surrey.

Sin embargo, la tesis de su enfermedad como causa del abandono del proyecto no resulta del todo plausible, dado que, como menciona en Deyan Sujdic en su biografía, Wendy no desarrolló la enfermedad hasta 1988, falleciendo en menos de un año.

\subsection{Un dilema}

Richard Horden también atribuye el abandono del proyecto a la persistencia en el estudio de un intenso debate, recurrente desde los tiempos del Team Four: expresar o no expresar los sistemas (estructura e instalaciones): el expresionismo del helicóptero Bell 47 D1, preferido por Richard Rogers, en el que se exhiben cada una de las piezas del conjunto y sus conexiones; frente a la envolvente aerodinámica del Bell Jet Ranger de Norman Foster, en el que la complejidad de los sistemas se encapsula en una forma escultural optimizada.

\footnotetext{
${ }^{30}$ Reyner Banham, "A home is not a house", Art in America, No. 2, abril 1965

31 Serge Chermayeff and Christopher Alexander. Community and Privacy: Toward a New Architecture of Humanism (London: Anchor Books, 1965), 64
} 
Este debate, que a priori puede parecer meramente formal, revela en realidad dos formas casi opuestas de abordar la complejidad de un edificio: la despreocupación formal de Richard Rogers y su interés por la capacidad expresiva de la estructura y las instalaciones, frente al rigor y la contención formal de Norman Foster. La exhibición del drama de las instalaciones mecánicas y del armazón estructural propia del Centro Pompidou, frente a la elegancia del contenedor neutro y monocromo del Sainsbury Centre. La preponderancia de la envolvente frente a la del esqueleto.

En la casa de los Foster, curiosamente más próxima a este segundo enfoque que al primero, la estructura adquiere progresivamente mayor importancia, exhibiéndose al exterior, hasta casi volverse excesiva y grotesca. El esmero artesanal con el que se moldean los perfiles y los complicados nudos estructurales en aluminio, remiten al preciosismo de las piezas en fundición diseñadas por Peter Rice para el Centro Pompidou. Una actitud que denota el alejamiento del planteamiento basado en la economía y la eficiencia propias de la "nave bien servida" y del kit de componentes de los Eames, para quienes la estructura no era más que un andamiaje neutro, con vocación de permanecer invisible.

Inmersos en tan intenso debate, cuando tras haber obtenido los permisos correspondientes, se completa la construcción de la base de hormigón, los Foster todavía no han tomado una decisión sobre cómo construir el contenedor ligero.

\section{5. "Fostersculpt"}

La casa de los Foster expresa el conflicto entre dos ideas antagónicas, ambas originadas en el campo del diseño industrial: el kit de componentes de los Eames y la artesanía industrial de Chareau y Bijvoet. La máquina no es sólo una metáfora: la vivienda misma adquiere la apariencia de una máquina.

Paradójicamente, aunque no pretende ser producida en serie, ni siquiera ser fabricada a partir de componentes estándar, ofrece el aspecto de un producto industrial susceptible de ser repetido. Aunque con toda probabilidad sus componentes nunca serán sustituidos ni reubicados en el transcurso de su vida útil, la configuración de la casa se justifica en base a esta remota posibilidad de transformación.

La casa no está concebida para ser transportada a otro lugar, ni tan solo para ser repetida en otro emplazamiento, pero simula levantarse levemente sobre el suelo, como esperando el día en que será desmontada y trasladada pieza por pieza a otro lugar. La obsesión por la ligereza, tan elocuentemente expresada por Buckminster Fuller en su famosa pregunta retórica sobre el peso del Sainsbury Centre, responde más a una voluntad expresiva que a una necesidad funcional.

El propio Norman Foster, interrogado 35 años después de que abandonara su proyecto en Hampstead reconoce que "habría que simplificarla mucho. Había mucho expresionismo estructural". Anthony Hunt, va más allá cuando afirma que "las primeras versiones de la casa, en acero, son mucho más eficientes, ya que están basadas en sistemas estándar, pueden ser repetidas y son Baratas. Pero el proyecto fue demasiado lejos".

Sentado en la RIBA Heinz Gallery junto al "Fostersculpt", ${ }^{32}$ un trozo del esqueleto de su casa no construida, con la que anuncia a los transeúntes el contenido de su exposición retrospectiva de 1979, Norman Foster parece adelantar su trayectoria futura, hacia una arquitectura de cada vez mayor sofisticación tecnológica que, sin embargo, no puede evitar reavivar cierta nostalgia a las propuestas utópicas de la década anterior. Una arquitectura, cuyo exhibicionismo estructural, traiciona los preceptos funcionalistas de sus primeras obras, al adoptar el repertorio formal de "la nave bien servida" para consolidar ese nuevo academicismo "heroico-tecnológico", que se ha dado en denominar High-Tech. Tal vez el "Fostersculpt" no esté tan alejado del neoclasicismo de la basa de la columna del edificio de Robert Adam, desde la que Norman Foster posa sonriente, pese a la incomodidad de su postura ¿Cabría pues sospechar que lo que se presenta es en realidad el agotamiento de una idea? ¿El final de trayecto de una "arquitectura del riesgo" que ya no arriesga? (Fig. 10)

\footnotetext{
32 "Foster at Home", Architect's Journal, No. 31, octubre 1979: 910-911.
} 


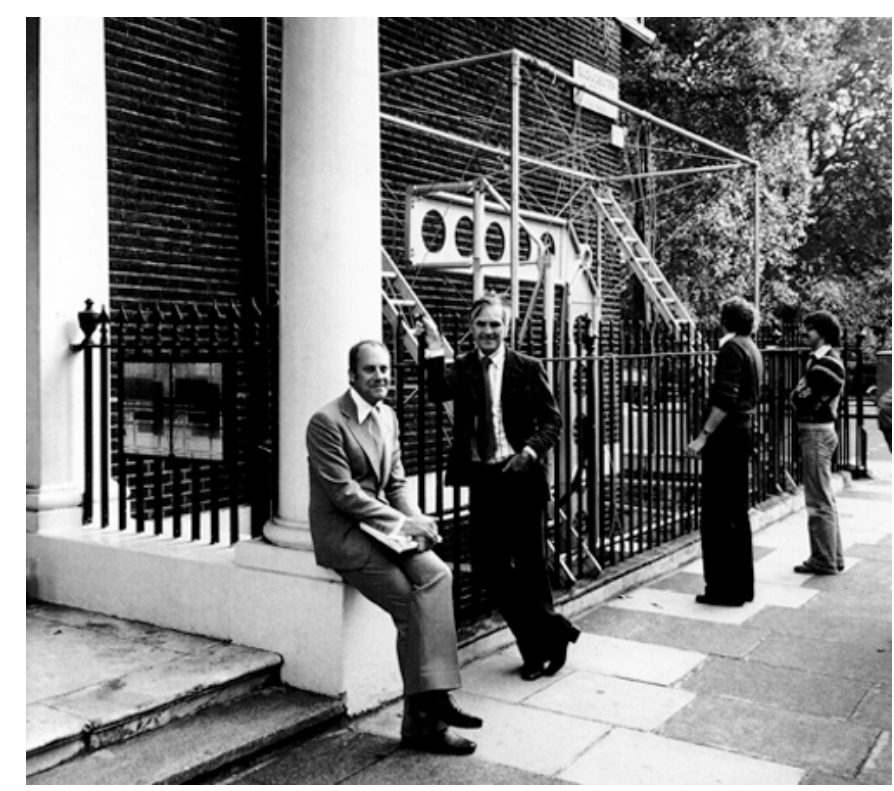

Fig. 10

\section{Imágenes}

Fig. 1: Croquis de Norman Foster para la fachada de la octava versión. (c) Foster + Partners.

Fig. 2: Croquis de Norman Foster para los componentes "enchufables" de la octava versión. @ Foster + Partners.

Fig. 3: Alzado para la octava versión, por Jan Kaplický. (c) Foster + Partners.

Fig. 4: Axonometría explotada para la octava versión, por Jan Kaplický. @ Foster + Partners.

Fig. 5: Croquis para la librería de la segunda versión, por Norman Foster. (c Foster + Partners.

Fig. 6: Planta y sección de la cuarta versión, por Richard Horden. (C) Foster + Partners.

Fig. 7: Planta y sección de la quinta versión, por Richard Horden. (c) Foster + Partners.

Fig. 8: Maqueta para la octava versión, por Chris Windsor. (C) Foster + Partners.

Fig. 9: Sección transversal de la octava versión, por Richard Horden y Jan Kaplický. (c) Foster + Partners.

Fig. 10: Norman Foster y John Harris junto a la entrada de la Heinz Gallery, Londres 1979. @ Foster + Partners.

\section{Bibliografía}

BACHMAN, Leonard R. Integrated Buildings: The Systems Basis of Architecture. Hoboken: John Wiley \& Sons, 2003. 480 p. ISBN: 0-471-38827-0.

BANHAM, Reyner. "A home is not a house", Art in America, No. 2, abril 1965. p. 109-118. ISSN: 0004-3214.

BANHAM, Reyner. Megastructure: Urban Futures of the Recent Past. London: Thames and Hudson, 1976. 224 p. ISBN: 0064303713.

BANHAM, Reyner. The Architecture of the Well-Tempered Environment. Chicago: The University of Chicago Press, 1969. 319p. ISBN: 978-0-85139-073-4.

BANHAM, Reyner. Theory and Design in the First Machine Age. London: Architectural Press, 1960. 338 p.

CHERMAYEFF, Serge, and ALEXANDER, Christopher. Community and Privacy: Toward a New Architecture of Humanism. London: Anchor Books, 1965. 255 p. ISBN: 0-385-03476-8.

DALE, Nigel. Connexions: The Unseen Hand of Tony Hunt. Dunbeath: Whittles Publishing, 2012. 198 p. ISBN: 978-184995-030-5.

DAVIES, Colin. High Tech Architecture. London: Thames and Hudson, 1991. 159 p. ISBN: 0-8478-0881-5.

EHRENKRANTZ, Ezra D. Architectural Systems. A Needs, Resources, and Design Approach. New York: Mc Graw-Hill, 1989.

Foster Associates: Introduction by Reyner Banham. London: RIBA Publications Ltd, 1979. 72 p. ISBN: 0900-630-74-4.

"Foster at Home." Architect's Journal 31, octubre 1979. p. 910-911. ISSN: 0003-8466.

FOSTER, Norman. Foster Associates Buildings and Projects Volume 2: 1971-1978. Edited by lan Lambot. Surrey: Watermark, 1989. 240 p. ISBN: 962-7274-02-X.

FOSTER, Norman. Norman Foster: Works 1. Jenkins, David (ed.). London: Prestel, 2002. 579 p. ISBN: 37913-2534-5.

HORDEN, Richard. Light Tech: Towards a Light Architecture. Blaser, Werner (ed.). Basel: Birkhauser, 1995. 180 p. ISBN: 3-7643-5220-5. 
MACDONALD, Angus. The Engineer's Contribution to Contemporary Architecture: Anthony Hunt. London: Thomas Telford Publishing, 2000. 163 p. ISBN: 0-7277-2769-9.

"Maison Test Rig." L'Architecture d'Aujourd'hui, 212, diciembre 1980. p. 72-73. ISSN: 0009-8695.

MOORE, Charles, ALLEN, Gerald and LYNDON, Donlyn. The Place of Houses. New York: Henry Holt \& Co, 1979. 315 p. ISBN: 978-0520223578.

RUSH, Richard D, ed. The Building Systems Integration Handbook. New York: John Wiley \& Sons, 1986. 445 p. ISBN: 0-471-86238-X.

"Test Rig House Re-Explores Panelization, Energy Use." Architectural Record, Mid-August 1979. p. 64-65. ISSN: 0002-858X.

\section{Biografía}

Carlos Solé Bravo es arquitecto por la Escuela Técnica Superior de Arquitectura del Vallés (ETSAV-UPC), Máster en Proyectos Arquitectónicos Avanzados por la Escuela Técnica Superior de Arquitectura de Madrid (ETSAM-UPM), donde cursa estudios de doctorado.

Actualmente es Associate Partner en Foster + Partners, estudio en el que trabaja desde el año 2002, en las oficinas de Londres, Madrid y Panamá. Ha trabajado en proyectos internacionales como la Torre Cepsa en Madrid, las torres Omkar Worli en Mumbai o el Aeropuerto Internacional de Tocumen en Panamá, entre otros.

\section{Biography}

Carlos Solé Bravo graduated as an architect at Escuela Técnica Superior de Arquitectura del Vallés (ETSAVUPC). He gained a Masters in Advanced Architectural Projects at Escuela Técnica Superior de Arquitectura de Madrid (ETSAM-UPM), where he is currently a PHD student.

$\mathrm{He}$ is an Associate Partner at Foster + Partners, where he works since 2002, at the offices in London, Madrid and Panamá. He has been involved in several international projects such as the Cepsa Tower in Madrid, the Omkar Worli Towers in Mumbai and Tocumen International Airport in Panama, amongst others. 